\title{
Reply to 'Impact of optical aberrations on axial position determination by photometry'
}

\author{
Christian Franke ${ }^{1}$ and Sebastian van de Linde ${ }^{2}$ \\ ${ }^{1}$ Max Planck Institute of Molecular Cell Biology and Genetics, Dresden, Germany. \\ ${ }^{2}$ Department of Physics, University of Strathclyde, Glasgow, UK \\ Email: cfranke@mpi-cbg.de, s.vandelinde@strath.ac.uk
}

Author's Accepted Manuscript; Published as C. Franke \& S. van de Linde, Nat. Methods 15, 990992 (2018), https://doi.org/10.1038/s41592-018-0228-3

Gaussian fitting is a widely used and fundamental tool in single-molecule localization microscopy (SMLM), but the intensity extraction from experimental data can be challenging. ${ }^{1}$ We appreciate the thorough work of Rieger et al. on proposing the principal role of small optical aberrations, present in any state-of-the-art microscope, in the model mismatch between commonly used Gaussian PSF fitting for SMLM and the experimental PSF, even in focus. ${ }^{2}$ In our original paper ${ }^{1}$, the simulated emission profile of an emitter was based on the experimental examination of a 'spot' as there is no obvious distinction between the tail of the spot and the continuum of the detector. We agree that any aperture based-concept will not be able to capture the total number of arriving photons onto the detector as the probability of detecting photons far away from the molecule's center of mass is not 0 (Supplementary Fig. 1). Moreover, in an experimental situation, i.e. in the presence of potentially inhomogeneous background, one cannot distinguish between singular photons arriving micrometers away from their respective center of origin and those from other emitting objects. Nevertheless, we observe that TRABI enables the accurate determination of the photon number along an increased axial range from the significant part of this pattern, i.e. the spot, in contrast to Gaussian fitting. ${ }^{1}$

In our original work we derived the full-width at half-maximum (FWHM) from single-molecule measurements and used a radius exceeding 1.86xFWHM for experimental data (Supplementary Fig. 1). Expanding the aperture for an isolated emitter might result in capturing more than $95 \%$ of the detected photons, but intensity estimation will then be impaired by typical single-molecule densities in real experiments due to overlapping apertures. The marginal fraction of intensity from peripheral photons, which are not contributing to the spot, might not be extractable anymore and constitute part of the background. As shown by Rieger et al., fitting a full vectorial PSF model to experimental bead data is superior to Gaussian fitting and yields different photon numbers than photometry. Though the actual TRABI approach was not employed in their study, 
as the background was determined in the periphery of non-blinking individual beads, thus resembling aperture photometry. ${ }^{1,2}$ Certainly, the practical implication, e.g. the usage of the vectorial fit for gaining axial information would be of great interest, as was shown in similar manner by fitting cubic spline interpolated PSF models. ${ }^{3}$

For 3D TRABI imaging, the determination of the totally detected photon distribution of a fluorophore is not compulsory. The method rather relies on determining intensities in two differently sized apertures, which can both be smaller than the spot size. This also applies to intensity-based biplane imaging, where the intensity ratio was measured from two ${ }^{1}$ or more ${ }^{4}$ apertures per image plane.

To further quantify the performance of TRABI we imaged a $80 \mathrm{~nm}$ 3D DNA origami PAINT nanoruler across a large field of view with calibrated TRABI (Fig. 1, Supplementary Methods). ${ }^{5}$ The 3D orientation of the nanoruler could be successfully resolved (Fig. 1b) with an axial localization precision of $32.1 \mathrm{~nm}$ (Fig. 1c). Using the $x$-y distance and the axial height (Fig. 1d) the nanoruler length could be determined to $80.1 \pm 15.9 \mathrm{~nm}$ (s.d.) (Fig. 1e), which is in excellent agreement with the theoretical value. Similar values were also obtained with intensity based biplane imaging (BP-TRABI) with increased precision (Supplementary Fig. 2). Finally, we monitored the nanoruler length and $z$-position across the entire field of view (Fig. 1f). The results demonstrate no significant impact of field-dependent aberrations on our measurements, albeit at a relatively small observed axial range limited by the nanoruler itself. Undoubtedly, when exploring samples at greater depths possible aberrations could have a bigger impact on the spot shape thus introducing greater errors. Such effects of aberrations are also relevant to a wide range of 3D SMLM methods. ${ }^{6}$ Nevertheless, high-quality axial position measurements are still possible with TRABI, especially the measurements of relative positions. We validated these results with classical biplane imaging and obtained similar results (Supplementary Figs. 3\&4), thus demonstrating that in our setup aberrations do not affect TRABI more than other methods. However, the overall quality of any 3D method will benefit when correcting for aberrations, ${ }^{6}$ though it might be worth considering if aberrations could also be advantageously exploited as recently demonstrated. ${ }^{3}$

\section{References}

1. Franke, C., Sauer, M., van de Linde, S. Nat. Methods 14, 41-4 (2017).

2. Thorsen, R. $\varnothing$., Hulleman, C. N., Stallinga S., Rieger B., submitted

3. Li, Y. et al. Nat. Methods 15, 367-9 (2018)

4. Velmurugan, R., Chao, J., Ram, S., Ward, E.S., Ober, R.J. Opt. Express 25, 3394-410 (2017)

5. Schmied et al. Nano Lett. 2, 781-785 (2013)

6. Booth, M., Andrade, D., Burke, D., Patton, B., Zurauskas, M. Microscopy (Oxf), 64, 251$61(2015)$ 


\section{Acknowledgement}

We are grateful to M. Sauer for support and D. Helmerich for technical assistance. We also thank R. Thorsen, S. Stallinga and B. Rieger for discussion.

\section{Conflict of interest}

The authors filed a patent with Carl Zeiss Microscopy GmbH (No. 102016014 133.6).

\section{Code availability}

TRABI version 1.2 runs in Image and is available from

http://bcp.phys.strath.ac.uk/photophysics/super-resolution/software/

\section{Data availability}

Data are available from the authors upon reasonable request. 

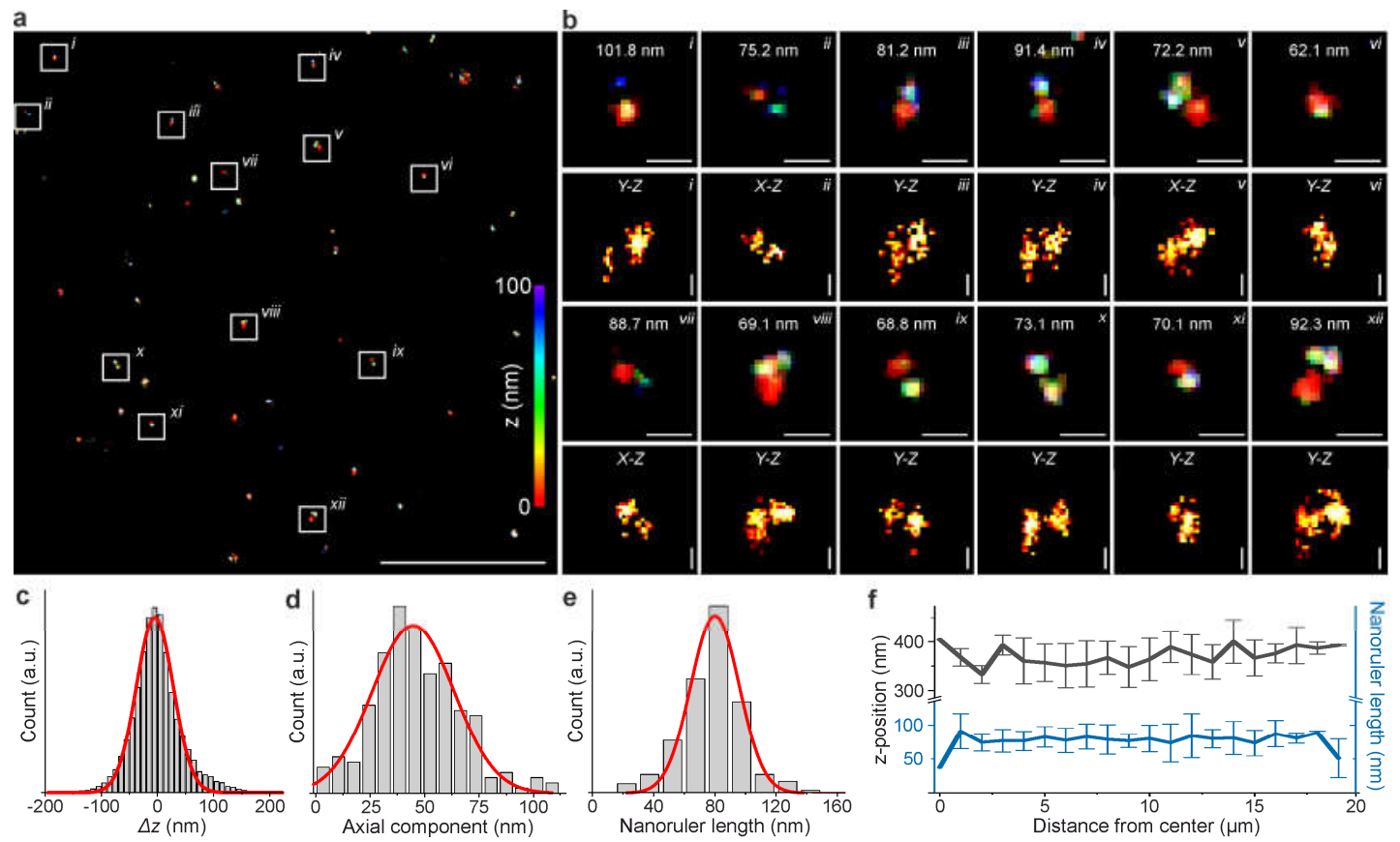

Fig. 1 3D TRABI imaging of a DNA origami sample consisting of two binding sites separated by 80 $\mathrm{nm}$. a) Representative part of a DNA-PAINT image and $\mathbf{b}$ ) magnified regions as indicated in a) showing $x-y$ and corresponding $x-z$ or $y-z$ projections. The numbers in $n m$ reflect the calculated length of the nanoruler. c) Axial TRABI localization precision, standard deviation of Gaussian fit = $32.1 \mathrm{~nm}$ (FWHM = 77.5 nm). d) Axial distance of the two spots, Gaussian fit yields $44.7 \pm 18.9 \mathrm{~nm}$ (mean \pm s.d.), median $=44.5 \mathrm{~nm}$. e) Distribution of the calculated nanoruler length, Gaussian fit yields $80.1 \pm 15.9 \mathrm{~nm}$ (mean \pm s.d.), median = $78.5 \mathrm{~nm}$. f) The absolute $z$-position of all localizations (black) and the nanoruler length (blue) as function of the distance from the center of the field of view (mean and s.d.). First and last data points are affected by a reduced sample size (Supplementary Table 1). Three independent field of views ( $34 \mu \mathrm{m} \times 34 \mu \mathrm{m}$ ) were analyzed. $\mathrm{n}=222$ single spots comprising 18,232 individual localization for c)- f). Scale bars, a) $2 \mu \mathrm{m}$, b) 100 $\mathrm{nm}$. 


\section{Supplementary Information}
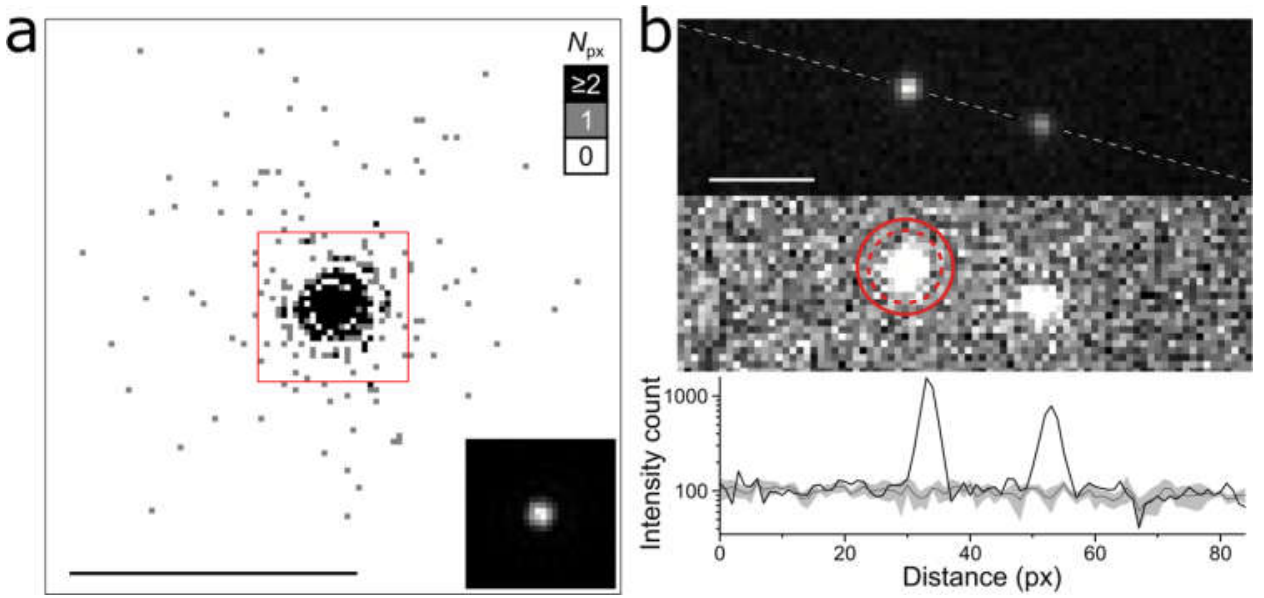

Supplementary Fig. 1 Theoretical emission profile and experimental spot. a) Simulated areal Airy-PSF emission pattern of 2,500 photon counts distributed over $10 \times 10 \mu \mathrm{m}^{2}$ without background ( $F W H M=340 \mathrm{~nm}$ ). Inverted image with $\geq 2$ photon count per pixel $\left(N_{\mathrm{px}}\right)$ saturation. Inset shows unsaturated partial image of the boxed region at the same scale, indicated in red. b) Top: experimental single-molecule image of Cy5 molecules under dSTORM conditions; middle: same region in saturation, experimental TRABI radius is shown as solid circle $(0.865 \mu \mathrm{m})$, $1.86 x$ FHM radius is shown as dashed circle $(0.632 \mu \mathrm{m})$; bottom: intensity profile in black as indicated in the top panel, the background mean and standard deviation from five consecutive frames is shown in gray and light gray, respectively. Scale bars, a) $5 \mu \mathrm{m}$, b) $2 \mu \mathrm{m}$. 

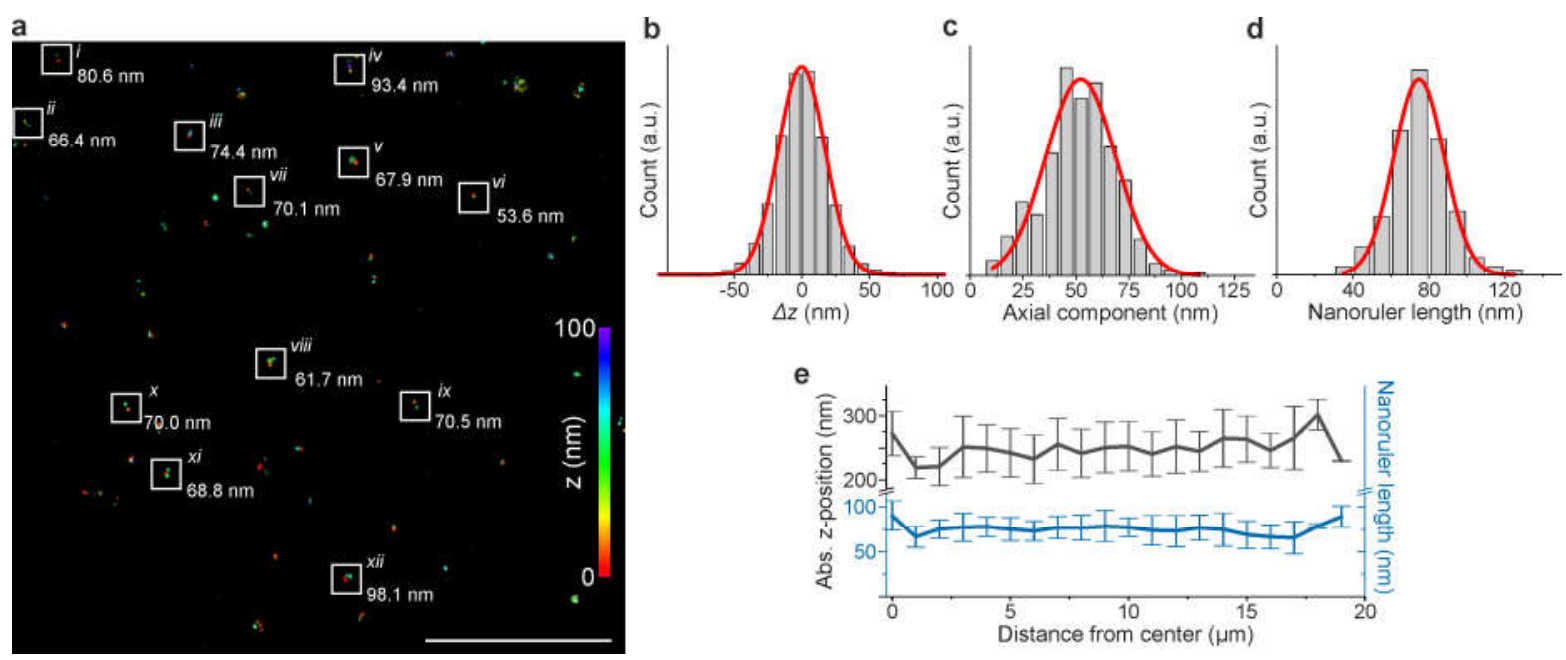

Supplementary Fig. 2 3D intensity based biplane imaging of the DNA origami sample. a) Representative part of a DNA-PAINT image. The numbers in $\mathrm{nm}$ reflect the calculated length of the selected nanoruler. $\mathbf{b})$ Axial localization precision of BP-TRABI, standard deviation of Gaussian fit $=17.2 \mathrm{~nm}$ (FWHM $=40.5 \mathrm{~nm}$ ). c) Axial distance of the two spots, Gaussian fit yields $52.3 \pm 16.5$ $\mathrm{nm}$ (mean \pm s.d.), median $=51.4 \mathrm{~nm}$. d) Distribution of the calculated nanoruler length, Gaussian fit yields $74.8 \pm 13.2 \mathrm{~nm}$ (mean \pm s.d.), median $=75.5 \mathrm{~nm}$. e) The absolute $z$-position of all localizations (black) and the nanoruler length (blue) as function of the distance from the center of the field of view (mean and s.d.). First and last data points are affected by a reduced sample size (Table S1). Three independent field of views (34 $\mu \mathrm{m} \times 34 \mu \mathrm{m})$ were conflated for data analysis. $n=383$ single spots comprising 40,091 individual localizations for b)- e) Scale bar, a) 2 $\mu \mathrm{m}$. 

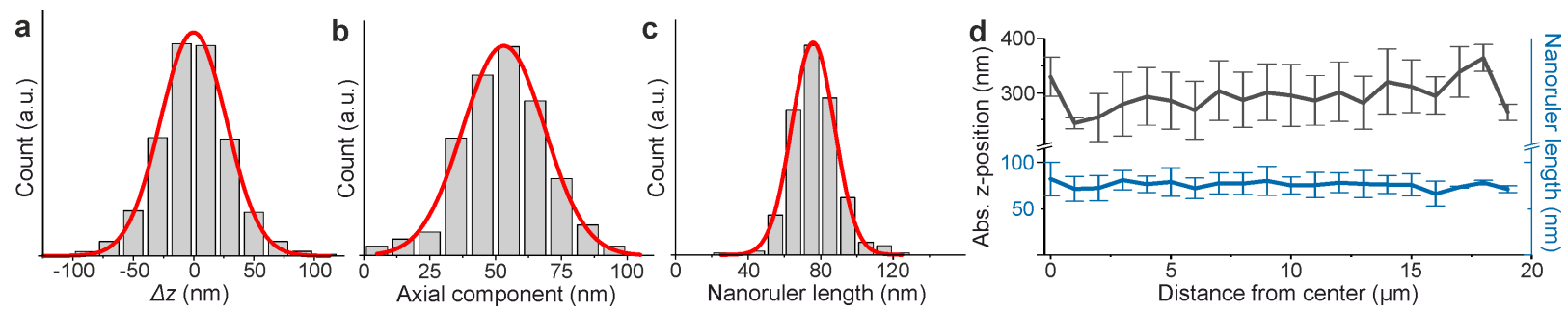

Supplementary Fig. 3 3D classical biplane imaging of the DNA origami sample. a) Axial localization precision of BP-FWHM, standard deviation of Gaussian fit $=26.8 \mathrm{~nm}$ (FWHM $=63.1$ $\mathrm{nm}$ ). b) Axial distance of the two spots, Gaussian fit yields $53.1 \pm 15.6 \mathrm{~nm}$ (mean \pm s.d.), median $=52.8 \mathrm{~nm}$. d) Distribution of the calculated nanoruler length, Gaussian fit yields $75.8 \pm 11.8 \mathrm{~nm}$ (mean \pm s.d.), median $=75.5 \mathrm{~nm}$. d) The absolute $z$-position of all localizations (black) and the nanoruler length (blue) as function of the distance from the center of the field of view (mean and s.d.). First and last data points are affected by a reduced sample size (Table S1). Three independent field of views ( $34 \mu \mathrm{m} \times 34 \mu \mathrm{m}$ ) were conflated for data analysis. $\mathrm{n}=353$ single spots comprising 41,928 individual localizations.

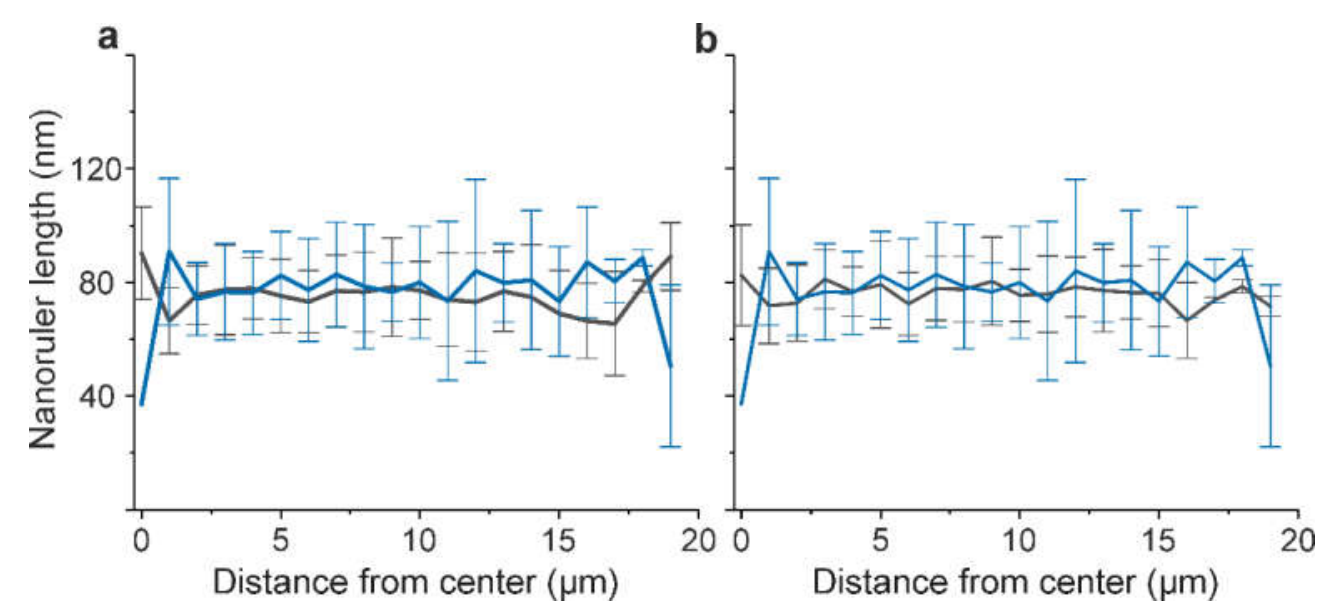

Supplementary Fig. 4 Field-dependent nanoruler length of different 3D methods in comparison. a) BP-TRABI (black, $n=383$ single spots comprising 40,091 individual localizations) vs. TRABI (blue, $n=222$ single spots comprising 18,232 individual localizations), b) Classical biplane imaging (BP-FWHM, black, $\mathrm{n}=353$ single spots comprising 41,928 individual localizations) vs. TRABI (blue). Displayed are mean value and s.d. (Supplementary Table 1). 
Supplementary Table 1. Sample size $(n)$ and statistics of DNA origami nanoruler length $(L)$ as function of the distance from the center of the field of view. The beginning and the end of the graph in Fig. 1f and Supplementary Figs. 2e, 3d, $\mathbf{4}$ are affected by a reduced sample size. On average $L$ varied in the range of $4 \%$ of the mean value $(4.0 \%$ for TRABI, 3.9\% for BP-TRABI, 3.4\% for BP-FWHM, without the first and last two bins).

\begin{tabular}{|c|c|c|c|c|c|c|c|c|c|}
\hline \multirow{2}{*}{$\underset{\text { binning }}{\mu \mathrm{m}}$} & \multicolumn{3}{|c|}{$L$ TRABI } & \multicolumn{3}{|c|}{$L$ BP-TRABI } & \multicolumn{3}{|c|}{ L BP-FWHM } \\
\hline & $n$ & mean $(\mathrm{nm})$ & s.d (nm) & $n$ & mean $(\mathrm{nm})$ & $\begin{array}{l}\text { s.d } \\
(\mathrm{nm})\end{array}$ & $n$ & mean $(\mathrm{nm})$ & s.d (nm) \\
\hline $0-1$ & 1 & 37.1 & 0.0 & 2 & 90.3 & 16.3 & 2 & 82.5 & 17.8 \\
\hline $1-2$ & 3 & 90.8 & 25.9 & 6 & 66.5 & 11.7 & 5 & 71.8 & 13.3 \\
\hline $2-3$ & 8 & 74.1 & 12.8 & 10 & 75.6 & 10.3 & 10 & 72.7 & 13.4 \\
\hline $3-4$ & 4 & 76.7 & 17.0 & 13 & 77.4 & 15.8 & 11 & 81.1 & 10.3 \\
\hline $4-5$ & 17 & 76.3 & 14.5 & 17 & 77.9 & 10.8 & 15 & 76.8 & 8.7 \\
\hline $5-6$ & 14 & 82.4 & 15.5 & 18 & 75.2 & 12.9 & 17 & 79.2 & 15.3 \\
\hline $6-7$ & 19 & 77.3 & 18.1 & 22 & 73.2 & 11.0 & 21 & 72.4 & 11.1 \\
\hline $7-8$ & 10 & 82.7 & 18.6 & 29 & 77.0 & 12.6 & 27 & 77.8 & 11.2 \\
\hline $8-9$ & 16 & 78.6 & 21.9 & 32 & 76.6 & 14.0 & 32 & 77.6 & 11.5 \\
\hline $9-10$ & 20 & 76.6 & 10.2 & 35 & 78.3 & 17.4 & 32 & 80.4 & 15.5 \\
\hline $10-11$ & 17 & 79.9 & 19.7 & 28 & 77.2 & 10.2 & 28 & 75.5 & 9.2 \\
\hline $11-12$ & 16 & 73.5 & 28.0 & 29 & 73.9 & 16.4 & 27 & 75.9 & 13.5 \\
\hline $12-13$ & 13 & 84.0 & 32.3 & 29 & 73.1 & 17.2 & 27 & 78.4 & 10.5 \\
\hline $13-14$ & 18 & 79.8 & 13.7 & 34 & 76.8 & 14.0 & 33 & 77.1 & 14.5 \\
\hline $14-15$ & 18 & 80.7 & 24.5 & 29 & 74.8 & 18.4 & 25 & 76.4 & 9.4 \\
\hline $15-16$ & 14 & 73.3 & 19.2 & 27 & 69.0 & 15.1 & 21 & 76.2 & 11.8 \\
\hline $16-17$ & 5 & 87.1 & 19.4 & 15 & 66.5 & 13.2 & 13 & 66.5 & 13.4 \\
\hline $17-18$ & 4 & 80.4 & 7.7 & 4 & 65.5 & 18.2 & 3 & 73.8 & 1.0 \\
\hline $18-19$ & 3 & 88.6 & 2.8 & 2 & 78.6 & 2.1 & 2 & 78.6 & 2.4 \\
\hline $19-20$ & 2 & 50.6 & 28.6 & 2 & 89.1 & 11.9 & 2 & 71.5 & 3.5 \\
\hline
\end{tabular}




\section{Material and Methods}

Setup. Measurements were performed on the same setup as described previously. ${ }^{1}$ The DNAorigami sample was imaged by DNA-PAINT in total internal reflection fluorescence (TIRF) mode. The incoming fluorescent light of ATT655 was split by a 50/50 beam splitter and imaged with two EMCCD cameras (IXON-Ultra 897, Andor) allowing to use single-plane TRABI and biplane imaging at the same time. The image splitter was used to allow a direct comparison between TRABI and biplane (Fig. 1, Supplementary Figs. 2\&3) but is not mandatory for single-plane TRABI. Leaving out the image splitter would have increased the photon yield of ATTO655 (on average 2,879 photons per spot and frame (median) as determined by TRABI).

TRABI and 3D analysis. TRABI version 1.2 (Ref. 2) was employed as described previously. ${ }^{1}$ Settings were $r_{1}=8.5 \mathrm{px}, \mathrm{BJ}=2, n_{\mathrm{BG}}=5, \mathrm{HF}=250$ frames, $P$ was determined using the intensities from fixed fitting in rapidSTORM 3.2 and the aperture formed by $r_{1}$.

Three 3D methods were employed, i) TRABI, ii) classical biplane (BP-FWHM) and iii) intensity based biplane (BP-TRABI) imaging. ${ }^{1}$ For every field of view, 20,000 frames were recorded with a frame rate of $50 \mathrm{~Hz}$. The analysis for BP-TRABI in Ref. 1 was modified in a way that only spot intensities from fixed fitting in rapidSTORM in two planes were used. The calibration was done with an Alexa Fluor 647 single-molecule surface under dSTORM conditions using a z-piezo scanner (Pifoc, Physik Instrumente, Germany). ${ }^{1}$ For each method the z-calibration was axially averaged in $20 \mathrm{~nm}$ steps and fitted with a polynomial to generate a lookup table with $0.25 \mathrm{~nm}$ precision. For the DNA-PAINT acquisition the focal plane was adjusted slightly into the coverslip. Then, the steep flank of the $z$-dependence was used for three-dimensional imaging above the coverslip. The resulting $P$ values were translated in absolute $z$-coordinates using the lookup table via linear interpolation. All $z$-coordinates were corrected for the refractive index mismatch as described previously. ${ }^{1}$ In general, it is important to perform calibration and measurements under similar conditions.

The axial precision was determined by separating the localizations of each spot in the nanoruler and aligning the localizations to their centre of mass. Then a histogram of all localizations (222 spots with 18,232 localizations for TRABI, 383 spots with 40,091 localizations for BP-TRABI, 353 spots with 41,928 localizations for BP-FWHM) was generated and fitted with a Gaussian function. The standard deviation of the Gaussian function was stated as axial localization precision in this work (Fig. 1c, Supplementary Figs. 2b\&3a). The lateral localization precision of all three approaches were determined to be in the range of 8-12 $\mathrm{nm}$ (s.d.).

Data analysis was performed with rapidSTORM, ImageJ, Python and Origin. 
DNA origami. The DNA origami nanoruler 'GATTA-PAINT 3D HiRes 80R Expert Line' was purchased from GATTAquant (Germany). It has the form of a pillar and consists of two binding sites for diffusing ATTO655 labeled oligonucleotides being separated by $80 \mathrm{~nm}$. One side of the pillar is anchored to the coverslip and shows a broad angular distribution with standing and tilted rulers. ${ }^{3,4}$ Consequently, the axial height of the two separated spots was smaller than the nanoruler length $(<80 \mathrm{~nm})$. Because the sample also contained DNA-PAINT based fiducial markers, the nanorulers were manually selected (several hundred in 3 independent measurements). The spot pairs of each nanoruler were then geometrically characterized. Therefore, the average $x-, y$ - and $z$-coordinates of each spot were determined (e.g. spot 1: $X_{1}, Y_{1}$, $Z_{1}$ ) in order to calculate the distances in $x, y$, and $z$ of the spot pair. Finally, the nanoruler length $(L)$ was calculated according to $L^{2}=\left(X_{1}-X_{2}\right)^{2}+\left(Y_{1}-Y_{2}\right)^{2}+\left(Z_{1}-Z_{2}\right)^{2}$.

Imaging the origami sample was challenging in two terms, i.e. the axial resolution capability of TRABI is in the range of $80 \mathrm{~nm}$ (Fig. 1c) and TRABI had to handle an increased background generated by diffusing labels. Consequently, the collected sample size is reduced compared to both biplane approaches and the axial component of the measured nanoruler length is slightly smaller. This is due to the fact that more laterally separated spot-pairs were selected since the axial resolution power of TRABI is inferior to BP-FWHM and BP-TRABI.

\section{References}

1. Franke, C., Sauer, M., van de Linde, S. Nat. Methods 14, 41-4 (2017).

2. http://bcp.phys.strath.ac.uk/photophysics/super-resolution/software/, 2018

3. Schmied et al. Nano Lett. 2, 781-785 (2013)

4. http://www.gattaquant.com/products/localization-based/gatta-paint-3d.html, 2018 